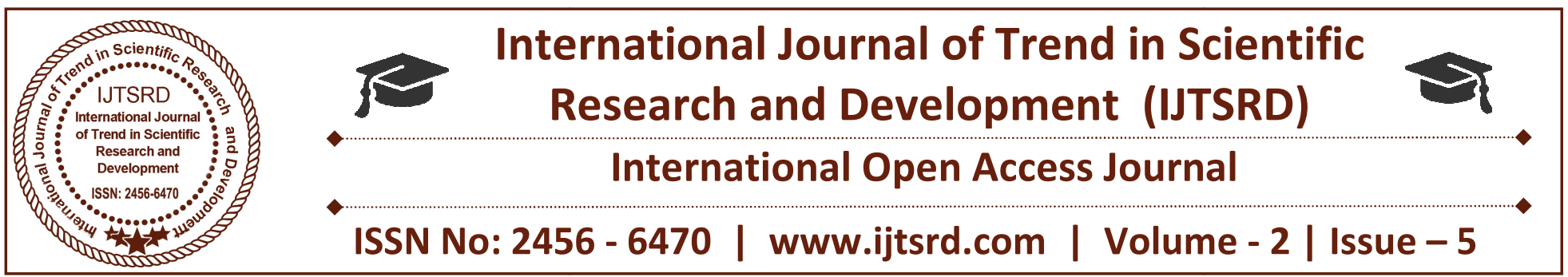

\title{
Memory Management in Operating System
}

\author{
Durgesh Raghuvanshi \\ B. Tech, Department of Computer Science, IILM Academy of Higher Learning, \\ Greater Noida, Uttar Pradesh, India
}

\begin{abstract}
In the recent era of computing, applications an operating system cannot survive without efficient memory management, especially if an application has to be under Surve load for an undefined long time. Resources must be utilized efficiently to enhance performance. This paper describes about the memory management in an operating system and it will demonstrate basic architecture of segmentation in an operating system and basic of its allocation. This paper also describes about the basic concept of virtual memory management and dynamic memory management.
\end{abstract}

\section{KEYWORD: Memory, Allocation, Segmentation,} Support in segmentation.

\section{INTRODUCTION}

An operating system (OS) is system software that manages computer hardware and software resources and provides common services for computer programs. All computer programs, excluding firmware, require an operating system to function.

The main purpose of a computer system to execute programs. These programs with their accusing data must locate in the main memory at the time for execution. The principles of managing the main memory which is one of the most precious resources in a multiprogramming system of an operating system.

Memory consists of a large array of words or bytes each with its own address. Modern operating systems provide efficient memory management and still research is being conducted to improve the way the allocated for applications because the main problem faces the memory allocation algorithm.
As we know that the goals of an operating system are convenient and efficiently so we should take care of these two goals of an operating system.

\section{Memory Management Schemes:}

Memory management Schemes are broadly divided into following categories:

\subsection{Contiguous Memory Allocation:}

Contiguous allocation means that each logical object is placed in a set of memory locations with strictly consecutive addresses. Bare machines, resident monitor, multiprogramming are in further division of contiguous allocation while multiprogramming is categorized into two parts i.e. Fixed partition and variable partition.

\subsection{Non-contiguous Memory Allocation:}

Non-contiguous allocation implies that a single logical object may be placed in non-consecutive sets of memory location. Paging and segmentation are the two mechanisms that are used to manage noncontiguous memory allocation. A better method to overcome the fragmentation problem was evolved which makes our logical address spaces noncontiguous in nature.

\section{Segmentation:}

Segmentation is another technique for the noncontiguous storage allocation. It is different from paging as pages are physical in nature and hence are of fixed size, whereas segments are logical divisions of a program and hence are of variable size.

It is a memory management scheme that supports the user view of memory rather than a system view of memory rather than system view memory as in paging, in which we are concerned with the division 
of physical memory into fixed size blocks called frames. In segmentation, we divide the logical address space into different segments. The size of a segment varies according to the data stored in it or the nature of operation performed on that segment.

\section{Paging:}

Paging is a memory management scheme that removes the requirement of contiguous allocation of physical memory. This scheme permits the physical address space of a process to be non-contiguous. Because of this advantage over the previous methods discussed for memory management, paging was used in most of the operating system.

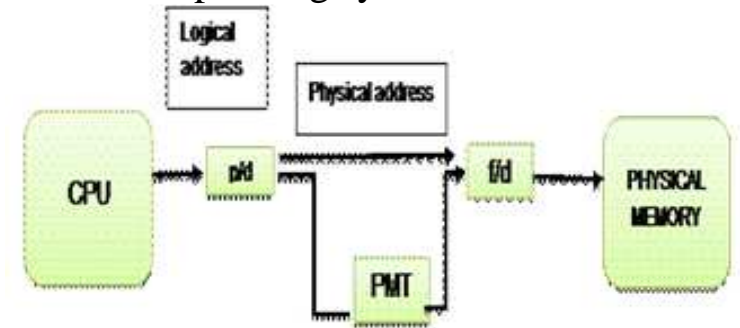

Paging System

\subsection{Demand paging:}

In demand paging a page is brought into the memory for its execution only when it is demanded otherwise it is remained in backing storage disk. The name seems to have been derived from demand feeding.

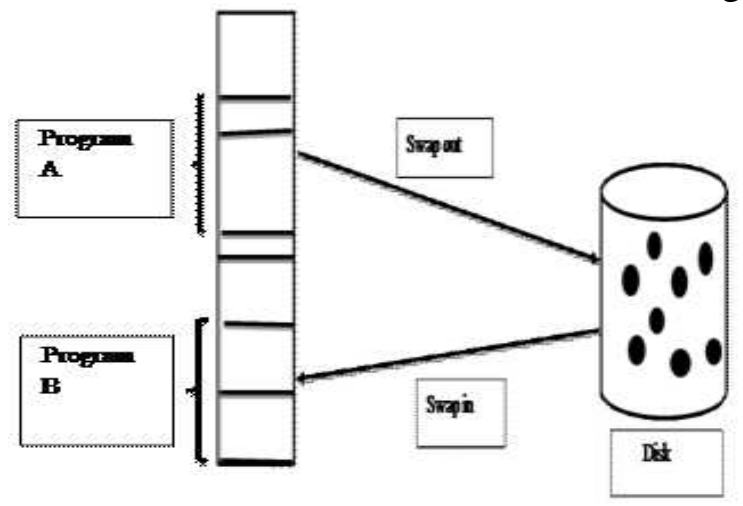

\section{Segmentation Architecture}

4.1. The logical address consists of a two tuple<segment-number, offset>

\subsection{Relocation}

4.2.1. Dynamic

4.2.2. By segment table

4.2.3. Sharing

4.2.4. Shared segments

4.2.5. Same segment number

4.2.6. Allocation
4.2.7. First bit/best fit

4.2.8. External fragmentation

4.3. Validation bit=0-illegal segment

4.4. Read, write / execute privileges.

\section{Virtual Memory:}

It is a virtual resource of a computer. It is an illusion that a system possesses more memory that is actually having this illusion makes a process independent of the size of real memory. It also permits a large number of processes to share a computer system without constraining each other.

The details of virtual memory management are generally transparent to programs. The ability to execute a partially loaded process is also advantageous from the operating system point of view, it also used to reduce the external fragmentation without the need to change the scheduled order of process executions.

\section{Hardware Implementation:}

In a system using segmentation, computer memory addresses consist of a segment id and an offset to the segment. A hardware memory management is responsible for translating the segment and offset into a physical memory address, and for performing checks to make sure the translation can be done and that the reference to that segment and offset is permitted.

\section{CONCLUSION}

Hence, with this we have understood about the indispensable concept of an operating system and its memory management and its segmentation in memory and also a short outlook of memory management.

In this paper different memory allocation techniques have been discussed along with their comparative analysis.

\section{REFERENCES}

1. Muhammad Abdullah Awais; MS150200157, Virtual University of Pakistan.

2. Martin Bohmert; Department of computer science University of Freiburg, Germany

3. Christoph Scholl; Department of computer science; University of Freiburg, Germany

4. https://whatis.techtarget.com/definition/segmented .memory

5. https://wikipedia.org/wiki/memory management, segmentation 